\title{
INTEGRALITY OVER FIXED RINGS OF AUTOMORPHISMS IN A LIE NILPOTENT SETTING
}

\author{
Jenő Szigeti
}

\begin{abstract}
Let $R$ be a Lie nilpotent algebra of index $k \geq 1$ over a field $K$ of characteristic zero. If $G$ is an $n$-element subgroup $G \subseteq \operatorname{Aut}_{K}(R)$ of $K$ automorphisms, then we prove that $R$ is right integral over $\operatorname{Fix}(G)$ of degree $n^{k}$. In the presence of a primitive $n$-th root of unity $e \in K$, for a $K$-automorphism $\delta \in \operatorname{Aut}_{K}(R)$ with $\delta^{n}=\operatorname{id}_{R}$, we prove that the skew polynomial algebra $R[w, \delta]$ is right integral of degree $n^{k}$ over $\operatorname{Fix}(\delta)\left[w^{n}\right]$.
\end{abstract}

\section{INTRODUCTION}

The notions of algebraicity and integrality play an important role in algebra and algebraic number theory. The aim of our paper is to present two integrality results, which are non-commutative generalizations of well known facts, the first one is closely related to classical Galois theory and the second one is related to the integrality of the commutative polynomial algebra $C[w]$ over $C\left[w^{n}\right]$.

A natural replacement of commutativity is the following: a ring $R$ is called Lie nilpotent of index $k$, if the left normed commutator product (with $[x, y]=x y-y x$ )

$$
\left[\left[\ldots\left[\left[x_{1}, x_{2}\right], x_{3}\right], \ldots, x_{k}\right], x_{k+1}\right]=0
$$

is a polynomial identity on $R$.

In section (5) we prove the following two integrality theorems.

Theorem (A). Let $R$ be a Lie nilpotent algebra of index $k \geq 1$ over a field $K$ of characteristic zero. If $G$ is an n-element subgroup $G \subseteq \operatorname{Aut}_{K}(R)$ of $K$ automorphisms, then $R$ is right integral over $\operatorname{Fix}(G)$ of degree $n^{k}$. In other words, for any $r \in R$ we have

$$
c_{0}+r c_{1}+\cdots+r^{n^{k}-1} c_{n^{k}-1}+r^{n^{k}}=0
$$

for some $c_{i} \in \operatorname{Fix}(G), 0 \leq i \leq n^{k}-1$.

Remarks to (A). Let $K \subseteq L$ be a field extension and $G \subseteq \operatorname{Aut}_{K}(L)$ be a finite subgroup of the $K$-automorphisms of $L$. Then $a \in L$ is a root of the monic polynomial $f(w)=\Pi_{\gamma \in G}(w-\gamma(a))$ of degree $n=|G|$ and the coefficients of $f(w) \in L[w]$ are in the fixed intermediate field $K \subseteq \operatorname{Fix}(G) \subseteq L$ of $G$. Thus $L$ is integral over

1991 Mathematics Subject Classification. 16R40,16S36,16S50,16W20,16W50.

The author was partially supported by the National Research, Development and Innovation. Office of Hungary (NKFIH) K119934. 
$\operatorname{Fix}(G)$ of degree $n$. We note that the replacement of $L$ by a commutative $K$-algebra $R$ (in the above argument) immediately yields Theorem (A) for $k=1$.

If $\operatorname{Fix}(G)=K$ and $a \in L$ is also a root of a $K$-irreducible polynomial $q(w) \in K[w]$, then $q(w)$ is a divisor of $f(w)$. For a finite normal field extension $K \subseteq L$, this observation shows that the Galois group $G=\operatorname{Aut}_{K}(L)$ acts transitively on the roots of $q(w)$.

Theorem (B). Let $R$ be a Lie nilpotent algebra of index $k \geq 1$ over a field $K$ of characteristic zero. If $e \in K$ is a primitive $n$-th root of unity and $\delta \in \operatorname{Aut}_{K}(R)$ is a $K$-automorphism with $\delta^{n}=\mathrm{id}_{R}$, then the skew polynomial algebra $R[w, \delta]$ is right integral of degree $n^{k}$ over the $K$-subalgebra $\operatorname{Fix}(\delta)\left[w^{n}\right]$ of $R[w, \delta]$. In other words, for any $f(w) \in R[w, \delta]$ we have

$$
g_{0}\left(w^{n}\right)+f(w) g_{1}\left(w^{n}\right)+\cdots+f^{n^{k}-1}(w) g_{n^{k}-1}\left(w^{n}\right)+f^{n^{k}}(w)=0
$$

for some $g_{i}\left(w^{n}\right) \in \operatorname{Fix}(\delta)\left[w^{n}\right], 0 \leq i \leq n^{k}-1$ (notice, that $w^{n}$ is central in $R[w, \delta]$ ).

Remarks to (B). For a commutative $K$-algebra $C$, the choice $R=C$ and $\delta=\operatorname{id}_{C}$ in Theorem (B) immediately yields the well-known fact, that the polynomial algebra $C[w]$ is integral of degree $n$ over the $C$-subalgebra $C\left[w^{n}\right]$ generated by the power $w^{n}$. A simple illustration of the case $n=2$ is the following: any $f(w) \in C[w]$ can be uniquely written as $f(w)=p\left(w^{2}\right)+w q\left(w^{2}\right)$ and $f^{2}(w)-2 p\left(w^{2}\right) f(w)+\left(p^{2}\left(w^{2}\right)-\right.$ $\left.w^{2} q^{2}\left(w^{2}\right)\right)=0$ proves the integrality of $C[w]$ over $C\left[w^{2}\right]$ of degree 2 .

The proofs of Theorem A and B are heavily based on the use of certain matrix algebras over the given Lie nilpotent algebra $R$. The so called Lie nilpotent CayleyHamilton theorem from [S1] is an indispensable ingredient of our development. In order to provide a self-contained treatment, we present the necessary prerequisites in sections (2),(3) and (4).

Throughout the paper an algebra $R$ means a not necessarily commutative unitary algebra over a field $K$ of characteristic zero, all $K$-subalgebras contain the identity and all $K$-endomorphisms preserve the identity. The group of units in $R$ is denoted by $\mathrm{U}(R)$ and the centre of $R$ is denoted by $\mathrm{Z}(R)$. The notation for the full $n \times n$ matrix algebra over $R$ is $\mathrm{M}_{n}(R)$ and $\mathrm{GL}_{n}(R)=\mathrm{U}\left(\mathrm{M}_{n}(R)\right)$. The matrix $E_{i, j} \in \mathrm{M}_{n}(R)$ has 1 in the $(i, j)$ position and zeros in all other positions. The fixed $K$-subalgebra of a $K$-endomorphism $\delta: R \longrightarrow R$ is $\operatorname{Fix}(\delta)=\{r \in R \mid \delta(r)=r\}$.

\section{THE ALGEBRA OF SKEW CENTRALIZING MATRICES}

Using the natural (element-wise) extension $\delta_{n}: \mathrm{M}_{n}(R) \longrightarrow \mathrm{M}_{n}(R)$ of a $K$ endomorphism $\delta: R \longrightarrow R$ and a matrix $W \in \mathrm{M}_{n}(R)$, one can define the subalgebra of skew $(\delta, W)$-centralizing matrices in $\mathrm{M}_{n}(R)$ as

$$
\mathrm{M}_{n}(R, \delta, W)=\left\{A \in \mathrm{M}_{n}(R) \mid \delta_{n}(A) W=W A\right\} .
$$

For the sake of brevity, in the rest of the paper we omit the qualifier "skew".

If $W \in \mathrm{M}_{n}(\operatorname{Fix}(\delta))$, then $\mathrm{M}_{n}(R, \delta, W)$ is closed with respect to the action of $\delta_{n}$.

Example (1). The natural $\mathbb{Z}_{2}$-grading $E=E_{0} \oplus E_{1}$ defines an automorphism $\varepsilon\left(g_{0}+g_{1}\right)=g_{0}-g_{1}$ of the Grassmann algebra

$$
\left.E=K\left\langle v_{1}, \ldots, v_{i}, \ldots\right| v_{i} v_{j}+v_{j} v_{i}=0 \text { for all } 1 \leq i \leq j\right\rangle
$$

generated by the infinite sequence of anticommutative indeterminates $\left(v_{i}\right)_{i \geq 1}$ 
with $v_{i}^{2}=0$.

Using the invertible diagonal matrix

$$
Q_{d}=E_{1,1}+\cdots+E_{d, d}-E_{d+1, d+1}-\cdots-E_{n, n}
$$

in $\mathrm{M}_{n}(K)$, we obtain the classical supermatrix algebra $\mathrm{M}_{n, d}(E)$ as

$$
\mathrm{M}_{n, d}(E)=\mathrm{M}_{n}\left(E, \varepsilon, Q_{d}\right)=\left\{A \in \mathrm{M}_{n}(E) \mid \varepsilon_{n}(A) Q_{d}=Q_{d} A\right\} .
$$

The shape of a matrix $A \in \mathrm{M}_{n, d}(E)$ is

$$
A=\left[\begin{array}{ll}
A_{1,1} & A_{1,2} \\
A_{2,1} & A_{2,2}
\end{array}\right]
$$

where the square blocks $A_{1,1}$ and $A_{2,2}$ are of sizes $d \times d$ and $(n-d) \times(n-d)$ and the rectangular blocks $A_{1,2}$ and $A_{2,1}$ are of sizes $d \times(n-d)$ and $(n-d) \times d$. The entries of $A_{1,1}$ and $A_{2,2}$ are in the even part $E_{0}$ of $E$, while the entries of $A_{1,2}$ and $A_{2,1}$ are in the odd part $E_{1}$ of $E$.

We note that $E$ is Lie nilpotent of index 2 , the $K$-algebras $\mathrm{M}_{n}(E)$ and $\mathrm{M}_{n, d}(E)$ play an important role in Kemer's classification of T-prime T-ideals (see [Ke]).

Example (2). A $\mathbb{Z}_{n}$-grading of $R$ is an $n$-tuple $\left(R_{0}, R_{1}, \ldots, R_{n-1}\right)$, where each $R_{i}$ is a $K$-subspace of $R$ such that

$$
R=R_{0} \oplus R_{1} \oplus \cdots \oplus R_{n-1}
$$

is a direct sum and $R_{i} R_{j} \subseteq R_{i+j}$ for all $i, j \in\{0,1, \ldots, n-1\}$, where $i+j$ is taken in $\{0,1, \ldots, n-1\}$ modulo $n$. Using such a $\mathbb{Z}_{n}$-grading of $R$, the $K$-subalgebra $\mathrm{M}_{n}^{g}(R)$ of $\mathrm{M}_{n}(R)$ is defined as follows:

$$
\begin{gathered}
\mathrm{M}_{n}^{g}(R)=\left\{A=\left[a_{i, j}\right] \in \mathrm{M}_{n}(R) \mid a_{i, j} \in R_{j-i} \text { for all } 1 \leq i, j \leq n\right\}= \\
{\left[\begin{array}{ccccc}
R_{0} & R_{1} & \cdots & R_{n-2} & R_{n-1} \\
R_{n-1} & R_{0} & R_{1} & \ddots & R_{n-2} \\
\vdots & R_{n-1} & \ddots & \ddots & \vdots \\
R_{2} & \ddots & \ddots & R_{0} & R_{1} \\
R_{1} & R_{2} & \cdots & R_{n-1} & R_{0}
\end{array}\right],}
\end{gathered}
$$

where $j-i$ is taken in $\{0,1, \ldots, n-1\}$ modulo $n$.

If $e \in K$ is a primitive $n$-th root of unity, then

$$
\widehat{e}\left(r_{0}+r_{1}+\cdots+r_{n-1}\right)=r_{0}+e r_{1}+\cdots+e^{n-1} r_{n-1}
$$

defines a $K$-automorphism $\widehat{e}: R \longrightarrow R$ of the $\mathbb{Z}_{n}$-graded $K$-algebra $R=R_{0} \oplus R_{1} \oplus$ $\cdots \oplus R_{n-1}$ and

$$
D_{e}=e^{-1} E_{1,1}+e^{-2} E_{2,2}+\cdots+e^{-n} E_{n, n}
$$

is a diagonal matrix in $\mathrm{GL}_{n}(K)$. Now $\mathrm{M}_{n}^{g}(R)$ can be obtained as the algebra of $\left(\widehat{e}, D_{e}\right)$-centralizing matrices:

$$
\mathrm{M}_{n}^{g}(R)=\mathrm{M}_{n}\left(R, \widehat{e}, D_{e}\right)=\left\{A \in \mathrm{M}_{n}(R) \mid \widehat{e}_{n}(A) D_{e}=D_{e} A\right\} .
$$

One of the remarkable properties of $(\delta, W)$-centralizing matrices is the following.

2.1. Proposition. If $W \in \mathrm{GL}_{n}(R) \cap \mathrm{M}_{n}(\mathrm{Z}(R))$ is an invertible matrix with central elements, then the trace of a matrix $A \in \mathrm{M}_{n}(R, \delta, W)$ is in the fixed ring of $\delta$, i.e. $\operatorname{tr}(A) \in \operatorname{Fix}(\delta)$. 
Proof. Since the entries of $W$ are central and $W^{-1} W=I_{n}$, a straightforward computation gives that $\operatorname{tr}\left(W A W^{-1}\right)=\operatorname{tr}(A)$ for all $A \in \mathrm{M}_{n}(R)$. Thus for a matrix $A \in \mathrm{M}_{n}(R, \delta, W)$ we have

$$
\delta(\operatorname{tr}(A))=\operatorname{tr}\left(\delta_{n}(A)\right)=\operatorname{tr}\left(W A W^{-1}\right)=\operatorname{tr}(A),
$$

whence $\operatorname{tr}(A) \in \operatorname{Fix}(\delta)$ follows.

Any $K$-endomorphism $\delta: R \longrightarrow R$ can be naturally extended to a $K$-endomorphism $\delta_{z}: R[z] \longrightarrow R[z]$ of the polynomial ring $R[z]$ : for $r_{0}, r_{1} \ldots, r_{t} \in R$ take

$$
\delta_{z}\left(r_{0}+r_{1} z+\cdots+r_{t} z^{t}\right)=\delta\left(r_{0}\right)+\delta\left(r_{1}\right) z+\cdots+\delta\left(r_{t}\right) z^{t} .
$$

The elements of the skew polynomial ring $R[w, \delta]$ in the skew indeterminate $w$ are left polynomials of the form $f(w)=r_{0}+r_{1} w+\cdots+r_{t} w^{t}$ with $r_{0}, r_{1}, \ldots, r_{t} \in R$. Besides the obvious addition, we have the following multiplication rule in $R[w, \delta]$ : $w r=\delta(r) w$. If $\delta^{n}=\operatorname{id}_{R}$ (such a $\delta$ is an automorphism), then $w^{n}$ is a central element of $R[w, \delta]$.

Let $G=\left\{\sigma_{1}, \sigma_{2}, \ldots, \sigma_{n}\right\} \subseteq \operatorname{Aut}_{K}(R)$ be an $n$-element subgroup of the group of all $K$-automorphisms of $R$ (with $\sigma_{n}=\operatorname{id}_{R}$ ). The fixed $K$-subalgebra of $G$ is

$$
\operatorname{Fix}(G)=\{r \in R \mid \sigma(r)=r \text { for all } \sigma \in G\}=\bigcap_{\sigma \in G} \operatorname{Fix}(\sigma) .
$$

Any element $\tau \in G$ defines a permutation $\pi \in \mathrm{S}_{n}$ by

$$
\tau \circ \sigma_{1}=\sigma_{\pi(1)}, \ldots, \tau \circ \sigma_{n}=\sigma_{\pi(n)}
$$

and the corresponding $n \times n$ permutation matrix is

$$
P_{\tau}=E_{1, \pi(1)}+\cdots+E_{n, \pi(n)} .
$$

2.2. Theorem. Let $G=\left\{\sigma_{1}, \sigma_{2}, \ldots, \sigma_{n}\right\} \subseteq \operatorname{Aut}_{K}(R)$ be an n-element subgroup of $K$-automorphisms. If $r \in R$, then

$$
\Gamma_{G}(r)=\sum_{i=1}^{n} \sigma_{i}(r) E_{i, i}
$$

defines a natural diagonal embedding $\Gamma_{G}: R \longrightarrow \mathrm{M}_{n}(R)$ of rings. For $r \in R$ and $c \in \operatorname{Fix}(G)$ we have $\Gamma_{G}(c r)=c \Gamma_{G}(r), \Gamma_{G}(r c)=\Gamma_{G}(r) c$ and $\Gamma_{G}(c)=c I_{n}$.

If $\tau \in G$, then $\Gamma_{G}: R \longrightarrow \mathrm{M}_{n}\left(R, \tau, P_{\tau}\right)$ is an embedding of $K$-algebras, where $P_{\tau} \in \mathrm{GL}_{n}(K)$ is the permutation matrix defined by $\tau$.

Proof. Since $\Gamma_{G}(r)$ is diagonal, the verification of the fact that $\Gamma_{G}$ is an injective ring homomorphism is straightforward.

If $r \in R$ and $c \in \operatorname{Fix}(G)$, then $\Gamma_{G}(c r)=c \Gamma_{G}(r), \Gamma_{G}(r c)=\Gamma_{G}(r) c$ and $\Gamma_{G}(c)=c I_{n}$ follow from the fact that $\sigma(c r)=c \sigma(r), \sigma(r c)=\sigma(r) c$ and $\sigma(c)=c$ for all $\sigma \in G$.

The calculations

$$
\tau_{n}\left(\Gamma_{G}(r)\right)=\sum_{i=1}^{n} \tau\left(\sigma_{i}(r)\right) E_{i, i}=\sum_{i=1}^{n} \sigma_{\pi(i)}(r) E_{i, i}
$$

and

$$
\begin{gathered}
\tau_{n}\left(\Gamma_{G}(r)\right) P_{\tau}=\left(\sum_{i=1}^{n} \sigma_{\pi(i)}(r) E_{i, i}\right)\left(\sum_{j=1}^{n} E_{j, \pi(j)}\right)=\sum_{i=1}^{n} \sigma_{\pi(i)}(r) E_{i, i} E_{i, \pi(i)}= \\
\sum_{i=1}^{n} \sigma_{\pi(i)}(r) E_{i, \pi(i)}=\sum_{i=1}^{n} E_{i, \pi(i)} \sigma_{\pi(i)}(r) E_{\pi(i), \pi(i)}= \\
\left(\sum_{i=1}^{n} E_{i, \pi(i)}\right)\left(\sum_{k=1}^{n} \sigma_{k}(r) E_{k, k}\right)=P_{\tau} \Gamma_{G}(r)
\end{gathered}
$$

show that $\Gamma_{G}(r) \in \mathrm{M}_{n}\left(R, \tau, P_{\tau}\right)$. 
2.3. Theorem. If $\delta: R \longrightarrow R$ is a K-endomorphism and $W \in \mathrm{M}_{n}(R)$, then any homomorphism $\varphi: R \longrightarrow \mathrm{M}_{n}(R, \delta, W)$ of $K$-algebras with $\varphi \circ \delta=\delta_{n} \circ \varphi$ has a unique $K$-homomorphic extension

$$
\varphi^{(\delta)}: R[w, \delta] \longrightarrow \mathrm{M}_{n}(R[z])
$$

such that $\varphi^{(\delta)}(r)=\varphi(r)$ and $\varphi^{(\delta)}(w)=W z$. For $\varphi^{(\delta)}$ we have

$$
\varphi^{(\delta)}\left(r_{0}+r_{1} w+\cdots+r_{t} w^{t}\right)=\varphi\left(r_{0}\right)+\varphi\left(r_{1}\right) W z+\cdots+\varphi\left(r_{t}\right) W^{t} z^{t} .
$$

(1) If $f(w) \in R[w, \delta]$ and $\varphi(c)=c I_{n}$ for some $c \in R$, then $\varphi^{(\delta)}(c f(w))=$ $c \varphi^{(\delta)}(f(w))$.

(2) If $\varphi$ is injective and $W \in \mathrm{GL}_{n}(R)$ is invertible, then $\varphi^{(\delta)}$ is also injective.

(3) If $W \in \mathrm{M}_{n}(\operatorname{Fix}(\delta))$, then $\varphi^{(\delta)}$ is an $R[w, \delta] \longrightarrow \mathrm{M}_{n}\left(R[z], \delta_{z}, W\right)$ homomorphism of $K$-algebras.

Proof. The $K$-linearity of $\varphi^{(\delta)}$ is clear. In order to see the multiplicative property of $\varphi^{(\delta)}$, it is enough to prove that

$$
\varphi^{(\delta)}\left(\left(r w^{i}\right)\left(s w^{j}\right)\right)=\varphi^{(\delta)}\left(r w^{i}\right) \varphi^{(\delta)}\left(s w^{j}\right)
$$

for all $r, s \in R$ and $0 \leq i, j$.

Using $\varphi \circ \delta=\delta_{n} \circ \varphi$ and $\varphi(s) \in \mathrm{M}_{n}(R, \delta, W)$ we obtain that $\varphi(\delta(s)) W=\delta_{n}(\varphi(s)) W$ $=W \varphi(s)$. We proceed by induction and assume that $\varphi\left(\delta^{i}(s)\right) W^{i}=W^{i} \varphi(s)$ holds for some $i \geq 1$. Now the substitution of $\delta(s)$ into the place of $s$ gives

$$
\varphi\left(\delta^{i+1}(s)\right) W^{i+1}=\varphi\left(\delta^{i}(\delta(s))\right) W^{i} W=W^{i} \varphi(\delta(s)) W=W^{i} W \varphi(s)=W^{i+1} \varphi(s) .
$$

Thus we have

$$
\begin{gathered}
\varphi^{(\delta)}\left(\left(r w^{i}\right)\left(s w^{j}\right)\right)=\varphi^{(\delta)}\left(r \delta^{i}(s) w^{i+j}\right)=\varphi\left(r \delta^{i}(s)\right) W^{i+j} z^{i+j}= \\
\varphi(r) \varphi\left(\delta^{i}(s)\right) W^{i} W^{j} z^{i+j}=\varphi(r) W^{i} \varphi(s) W^{j} z^{i+j}= \\
\varphi(r) W^{i} z^{i} \varphi(s) W^{j} z^{j}=\varphi^{(\delta)}\left(r w^{i}\right) \varphi^{(\delta)}\left(s w^{j}\right) .
\end{gathered}
$$

(1) It follows from $\varphi\left(c r_{i}\right)=c \varphi\left(r_{i}\right)$.

(2) It follows from the fact, that $\varphi\left(r_{i}\right) W^{i}=0$ implies $r_{i}=0$.

(3) The application of $\delta_{n} \circ \varphi=\varphi \circ \delta$ and $\delta_{n}(W)=W$ gives

$$
\begin{gathered}
\left(\delta_{z}\right)_{n}\left(\varphi^{(\delta)}(w)\right) W=\left(\delta_{z}\right)_{n}(\varphi(r) W z) W=\delta_{n}(\varphi(r) W) z W= \\
\delta_{n}(\varphi(r)) \delta_{n}(W) z W=\varphi(\delta(r)) W W z=W \varphi(r) W z=W \varphi^{(\delta)}(w),
\end{gathered}
$$

whence $\varphi^{(\delta)}(w) \in \mathrm{M}_{n}\left(R[z], \delta_{z}, W\right)$ follows. Since $\varphi^{(\delta)}\left(r_{i}\right)=\varphi\left(r_{i}\right) \in \mathrm{M}_{n}\left(R[z], \delta_{z}, W\right)$, we obtain that $\varphi^{(\delta)}(f(w)) \in \mathrm{M}_{n}\left(R[z], \delta_{z}, W\right)$ for all $f(w) \in R[w, \delta]$.

2.4. Corollary. For an automorphism $\delta \in \operatorname{Aut}_{K}(R)$ with $\delta^{n}=\operatorname{id}_{R}$, the number of elements of the cyclic subgroup $\langle\delta\rangle=\left\{\delta^{i} \mid 1 \leq i \leq n\right\}$ of $\operatorname{Aut}_{K}(R)$ is a divisor of $n$ (and equality not necessarily holds). A natural diagonal embedding $\Delta: R \longrightarrow$ $\mathrm{M}_{n}(R)$ of $K$-algebras can be defined by $\Delta(r)=\sum_{i=1}^{n} \delta^{i}(r) E_{i, i}$, where $r \in R$. Using $H=E_{1,2}+E_{2,3}+\cdots+E_{n-1, n}+E_{n, 1}$, a straightforward calculation shows that $\delta_{n}(\Delta(r)) H=H \Delta(r)$. It follows that $\Delta$ is an $R \longrightarrow \mathrm{M}_{n}(R, \delta, H)$ map. Since $\Delta \circ \delta=\delta_{n} \circ \Delta$ and $H \in \mathrm{GL}_{n}(K) \cap \mathrm{M}_{n}(\operatorname{Fix}(\delta))$, there is a unique homomorphic extension $\Delta^{(\delta)}: R[w, \delta] \longrightarrow \mathrm{M}_{n}(R[z])$ of $\Delta$ such that $\Delta^{(\delta)}(r)=\Delta(r), \Delta^{(\delta)}(w)=$ $\mathrm{Hz}$ and

$$
\Delta^{(\delta)}\left(r_{0}+r_{1} w+\cdots+r_{t} w^{t}\right)=\Delta\left(r_{0}\right)+\Delta\left(r_{1}\right) H z+\cdots+\Delta\left(r_{t}\right) H^{t} z^{t} .
$$


This map is a

$$
\Delta^{(\delta)}: R[w, \delta] \longrightarrow \mathrm{M}_{n}\left(R[z], \delta_{z}, H\right)
$$

embedding of $K$-algebras. If $c \in \operatorname{Fix}(\delta)$, then $\Delta^{(\delta)}(c f(w))=c \Delta^{(\delta)}(f(w))$ for all $f(w) \in R[w, \delta]$. Now $c H=H c$ ensures that $\Delta^{(\delta)}(f(w) c)=\Delta^{(\delta)}(f(w)) c$.

If $|\langle\delta\rangle|=n$, then the choice $G=\left\{\sigma_{1}=\delta, \sigma_{2}=\delta^{2}, \ldots, \sigma_{n}=\delta^{n}\right\}$ in Theorem 2.2 gives that $\Delta=\Gamma_{G}$ and $H=P_{\delta}$.

\section{THE LIE NILPOTENT CAYLEY-HAMILTON THEOREM}

A Lie nilpotent analogue of classical determinant theory was developed in [S1], further details can be found in [Do,SvW]. Here we present the basic definitions and results about the sequences of right determinants and right characteristic polynomials, including the so-called Lie nilpotent right Cayley-Hamilton identities.

For an $n \times n$ matrix $A=\left[a_{i, j}\right]$ over an arbitrary (possibly non-commutative) ring or algebra $R$ with 1 , the element

$$
\operatorname{sdet}(A)=\sum_{\tau, \pi \in \mathrm{S}_{n}} \operatorname{sgn}(\pi) a_{\tau(1), \pi(\tau(1))} \cdots a_{\tau(t), \pi(\tau(t))} \cdots a_{\tau(n), \pi(\tau(n))}
$$

of $R$ is called the symmetric determinant of $A$. The symmetric adjoint $A^{*}=\left[a_{r, s}^{*}\right]$ of $A=\left[a_{i, j}\right]$ is defined as the following natural symmetrization of the classical adjoint:

$$
a_{r, s}^{*}=\sum_{\tau, \pi} \operatorname{sgn}(\pi) a_{\tau(1), \pi(\tau(1))} \cdots a_{\tau(s-1), \pi(\tau(s-1))} a_{\tau(s+1), \pi(\tau(s+1))} \cdots a_{\tau(n), \pi(\tau(n))}
$$

where the sum is taken over all $\tau, \pi \in \mathrm{S}_{n}$ with $\tau(s)=s$ and $\pi(s)=r$. We note that the $(r, s)$ entry of $A^{*}$ is exactly the signed symmetric determinant $(-1)^{r+s} \operatorname{sdet}\left(A_{s, r}\right)$ of the $(n-1) \times(n-1)$ minor $A_{s, r}$ of $A$ arising from the deletion of the $s$-th row and the $r$-th column of $A$. If $R$ is commutative, then $\operatorname{sdet}(A)=n ! \operatorname{det}(A)$ and $A^{*}=(n-1) ! \operatorname{adj}(A)$, where $\operatorname{det}(A)$ and $\operatorname{adj}(A)$ denote the ordinary determinant and adjoint of $A$.

The next result of Domokos plays a fundamental role in the proof of Theorem 4.1, on which the rest of section (4) is based.

3.1. Theorem ([Do]]). Let $R$ be an algebra over a field $K$ of characteristic zero. If $A \in \mathrm{M}_{n}(R)$ and $T \in \mathrm{GL}_{n}(K)$, then $\left(T^{-1} A T\right)^{*}=T^{-1} A^{*} T$.

The right adjoint sequence $\left(P_{k}\right)_{k \geq 1}$ of a matrix $A \in \mathrm{M}_{n}(R)$ is defined by the recursion: $P_{1}=A^{*}$ and $P_{k+1}=\left(A P_{1} \cdots P_{k}\right)^{*}$ for $k \geq 1$. It is easy to see that for any $i \geq 1$, the right adjoint sequence of the matrix $A P_{1} \cdots P_{i}$ is $\left(P_{k}\right)_{k \geq i+1}$. The $k$-th right adjoint of $A$ is defined as

$$
\operatorname{radj}_{(k)}(A)=n P_{1} \cdots P_{k}
$$

The $k$-th right determinant of $A$ is the trace of $A P_{1} \cdots P_{k}$ :

$$
\operatorname{rdet}_{(k)}(A)=\operatorname{tr}\left(A P_{1} \cdots P_{k}\right) \text {. }
$$

We note that

$$
\operatorname{rdet}_{(1)}(A)=\operatorname{tr}\left(A A^{*}\right)=\operatorname{sdet}(A)=\operatorname{tr}\left(A^{*} A\right)
$$

and the following theorem shows that $\operatorname{radj}_{(k)}(A)$ and $\operatorname{rdet}_{(k)}(A)$ can play a role similar to that played by the ordinary adjoint and determinant in the commutative case. 
3.2. Theorem ([S1], [SvW]). If $\frac{1}{n} \in R$ and the ring $R$ is Lie nilpotent of index $k$, then for a matrix $A \in \mathrm{M}_{n}(R)$ we have

$$
\operatorname{Aradj}_{(k)}(A)=n A P_{1} \cdots P_{k}=\operatorname{rdet}_{(k)}(A) I_{n} .
$$

The above Theorem 3.2 is not used explicitly, however it helps our understanding and serves as a starting point in the proof of Theorem 3.4.

Let $R[x]$ denote the ring of polynomials of the single commuting indeterminate $x$, with coefficients in $R$. The $k$-th right characteristic polynomial of $A$ is the $k$-th right determinant of the $n \times n$ matrix $x I_{n}-A$ in $\mathrm{M}_{n}(R[x])$ :

$$
p_{A, k}(x)=\operatorname{rdet}_{(k)}\left(x I_{n}-A\right) \text {. }
$$

3.3. Proposition ([SvW]). The $k$-th right characteristic polynomial $p_{A, k}(x) \in R[x]$ of $A \in \mathrm{M}_{n}(R)$ is of the form

$$
p_{A, k}(x)=\lambda_{0}^{(k)}+\lambda_{1}^{(k)} x+\cdots+\lambda_{n^{k}-1}^{(k)} x^{n^{k}-1}+\lambda_{n^{k}}^{(k)} x^{n^{k}},
$$

where $\lambda_{0}^{(k)}, \lambda_{1}^{(k)}, \ldots, \lambda_{n^{k}-1}^{(k)}, \lambda_{n^{k}}^{(k)} \in R$ and $\lambda_{n^{k}}^{(k)}=n\{(n-1) !\}^{1+n+n^{2}+\cdots+n^{k-1}}$.

The degree and the leading coefficient of the $k$-th right characteristic polynomial in Proposition 3.3 will provide the degree of right integrality in Theorems (A) and $(\mathrm{B})$.

3.4. Theorem ([S1],[SvW]). If $\frac{1}{n} \in R$ and the ring $R$ is Lie nilpotent of index $k$, then a right Cayley-Hamilton identity

$$
\text { (A) } p_{A, k}=I_{n} \lambda_{0}^{(k)}+A \lambda_{1}^{(k)}+\cdots+A^{n^{k}-1} \lambda_{n^{k}-1}^{(k)}+A^{n^{k}} \lambda_{n^{k}}^{(k)}=0
$$

with right scalar coefficients holds for $A \in \mathrm{M}_{n}(R)$. We also have $(A) u=0$, where $u(x)=p_{A, k}(x) h(x)$ and $h(x) \in R[x]$ is arbitrary.

\section{THE RIGHT CHARACTERISTIC POLYNOMIALS OF A SKEW CENTRALIZING MATRIX}

4.1. Theorem. Let $R$ be an algebra over a field $K$ of characteristic zero, $\delta: R \longrightarrow R$ be a $K$-endomorphism and $W \in \mathrm{GL}_{n}(K)$ be an invertible matrix. If $A \in \mathrm{M}_{n}(R, \delta, W)$ is a $(\delta, W)$-centralizing matrix, then $A^{*} \in \mathrm{M}_{n}(R, \delta, W)$. In other words, the matrix algebra $\mathrm{M}_{n}(R, \delta, W)$ is closed with respect to taking the symmetric adjoint.

Proof. The definition of $A^{*}$ and the element-wise action of $\delta_{n}$ ensure that $\delta_{n}\left(A^{*}\right)=$ $\delta_{n}(A)^{*}$ for all $A \in \mathrm{M}_{n}(R)$. Since $W \in \mathrm{GL}_{n}(K)$, Theorem 3.1 gives that

$\left(W A W^{-1}\right)^{*}=W A^{*} W^{-1}$ for all $A \in \mathrm{M}_{n}(R)$. Thus for a matrix $A \in \mathrm{M}_{n}(R, \delta, W)$ we have

whence $A^{*} \in \mathrm{M}_{n}(R, \delta, W)$ follows.

$$
\delta_{n}\left(A^{*}\right)=\delta_{n}(A)^{*}=\left(W A W^{-1}\right)^{*}=W A^{*} W^{-1},
$$

Remark. If $A \in \mathrm{M}_{n, d}(E)=\mathrm{M}_{n}\left(E, \varepsilon, Q_{d}\right)$ as in Example (1), then the application of Theorem 4.1 gives that $A^{*} \in \mathrm{M}_{n, d}(E)=\mathrm{M}_{n}\left(E, \varepsilon, Q_{d}\right)$. This is one of the main results in $[\mathrm{S} 2]$. 
4.2. Theorem. Let $R$ be an algebra over a field $K$ of characteristic zero, $\delta: R \longrightarrow R$ be a $K$-endomorphism and $W \in \mathrm{GL}_{n}(K)$ be an invertible matrix. If $A \in \mathrm{M}_{n}(R, \delta, W)$ is a $(\delta, W)$-centralizing matrix and $k \geq 1$ is an integer, then for the $k$-th right determinant we have $\operatorname{rdet}_{(k)}(A) \in \operatorname{Fix}(\delta)$.

Proof. The repeated application of Theorem 4.1 gives that the recursion $P_{1}=A^{*}$ and $P_{k+1}=\left(A P_{1} \cdots P_{k}\right)^{*}$ starting from a $(\delta, W)$-centralizing matrix $A \in \mathrm{M}_{n}(R, \delta, W)$ defines a sequence $\left(P_{k}\right)_{k \geq 1}$ in $\mathrm{M}_{n}(R, \delta, W)$. Since $\operatorname{rdet}_{(k)}(A)=$ $\operatorname{tr}\left(A P_{1} \cdots P_{k}\right)$ is the trace of $A P_{1} \cdots P_{k} \in \mathrm{M}_{n}(R, \delta, W)$ and the trace of a $(\delta, W)$ centralizing matrix is in $\operatorname{Fix}(\delta)$ by Proposition 2.1, the proof is complete.

4.3. Corollary. Let $R$ be an algebra over a field $K$ of characteristic zero, $\delta: R \longrightarrow R$ be a $K$-endomorphism and $W \in \mathrm{GL}_{n}(K)$ be an invertible matrix. If $A \in \mathrm{M}_{n}(R, \delta, W)$ is a $(\delta, W)$-centralizing matrix and $k \geq 1$ is an integer, then for the $k$-th right characteristic polynomial we have $p_{A, k}(x) \in \operatorname{Fix}(\delta)[x]$. In other words, the coefficients of the $k$-th right $p_{A, k}(x)=\operatorname{rdet}_{(k)}\left(x I_{n}-A\right)$ characteristic polynomial are in $\operatorname{Fix}(\delta)$.

Proof. We use the natural extension $\delta_{x}: R[x] \longrightarrow R[x]$ of $\delta$. Since

$$
x I_{n}-A \in \mathrm{M}_{n}\left(R[x], \delta_{x}, W\right),
$$

Theorem 4.2 gives that $p_{A, k}(x)=\operatorname{rdet}_{(k)}\left(x I_{n}-A\right)$ is in $\operatorname{Fix}\left(\delta_{x}\right)=\operatorname{Fix}(\delta)[x]$.

Remark. If $A \in \mathrm{M}_{n, d}(E)=\mathrm{M}_{n}\left(E, \varepsilon, Q_{d}\right)$ as in Example (1), then the application of Corollary 4.3 gives that $p_{A, 2}(x) \in \operatorname{Fix}(\varepsilon)[x]=E_{0}[x]$. This fact was exploited in [S2].

4.4. Corollary. Let $R=R_{0} \oplus R_{1} \oplus \cdots \oplus R_{n-1}$ be a $\mathbb{Z}_{n}$-graded algebra over a field $K$ of characteristic zero and $e \in K$ be a primitive $n$-th root of unity. If $k \geq 1$ is an integer and $A \in \mathrm{M}_{n}^{g}(R)$ is a "graded" $n \times n$ matrix with respect to the given $\mathbb{Z}_{n}$-grading, then for the $k$-th right determinant and $k$-th right characteristic polynomial of $A$ we have

$$
\operatorname{rdet}_{(k)}(A) \in R_{0} \text { and } p_{A, k}(x)=\operatorname{rdet}_{(k)}\left(x I_{n}-A\right) \in R_{0}[x] .
$$

Proof. Since $\mathrm{M}_{n}^{g}(R)=\mathrm{M}_{n}\left(R, \widehat{e}, D_{e}\right)$ (see Example $\left.(2)\right)$ and Fix $(\widehat{e})=R_{0}$, Theorem 4.2 and Corollary 4.3 can be applied.

Remark. In the absence of a primitive $n$-th root of unity, the direct proof (not using Example (2), Theorem 4.2 and Corollary 4.3) of the implications

$A \in \mathrm{M}_{n}^{g}(R) \Longrightarrow A^{*} \in \mathrm{M}_{n}^{g}(R), \operatorname{rdet}_{(k)}(A) \in R_{0}, p_{A, k}(x)=\operatorname{rdet}_{(k)}\left(x I_{n}-A\right) \in R_{0}[x]$ is rather technical.

The combination of Theorem 3.4 and Corollary 4.3 gives the following.

4.5. Theorem. Let $R$ be an algebra over a field $K$ of characteristic zero, $\delta: R \longrightarrow R$ be an endomorphism and $W \in \mathrm{GL}_{n}(K)$ be an invertible matrix. If $R$ is Lie nilpotent of index $k$ and $A \in \mathrm{M}_{n}(R, \delta, W)$, then a right Cayley-Hamilton identity

$$
\text { (A) } p_{A, k}=I_{n} \lambda_{0}^{(k)}+A \lambda_{1}^{(k)}+\cdots+A^{n^{k}-1} \lambda_{n^{k}-1}^{(k)}+A^{n^{k}} \lambda_{n^{k}}^{(k)}=0
$$


holds, where the coefficients $\lambda_{i}^{(k)}, 0 \leq i \leq n^{k}$ of $p_{A, k}(x)=\operatorname{rdet}_{(k)}\left(x I_{n}-A\right)$ are in $\operatorname{Fix}(\delta)$. Since $\lambda_{n^{k}}^{(k)}=n\{(n-1) !\}^{1+n+n^{2}+\cdots+n^{k-1}}$ is invertible (in $K$ ), the above identity provides the right integrality of $\mathrm{M}_{n}(R, \delta, W)$ over $\operatorname{Fix}(\delta)$ of degree $n^{k}$.

\section{THE PROOFS OF THEOREM (A) AND (B)}

Proof of Theorem (A). Take an element $\tau \in G$ and consider the embedding $\Gamma_{G}: R \longrightarrow \mathrm{M}_{n}\left(R, \tau, P_{\tau}\right)$ in Theorem 2.2. For $r \in R$ Theorem 4.5 ensures that

$$
I_{n} \lambda_{0}^{(k)}+\Gamma_{G}(r) \lambda_{1}^{(k)}+\cdots+\left(\Gamma_{G}(r)\right)^{n^{k}-1} \lambda_{n^{k}-1}^{(k)}+\left(\Gamma_{G}(r)\right)^{n^{k}} \lambda_{n^{k}}^{(k)}=0
$$

holds for the $\left(\tau, P_{\tau}\right)$-centralizing matrix $\Gamma_{G}(r) \in \mathrm{M}_{n}\left(R, \tau, P_{\tau}\right)$, where

$$
p_{\Gamma_{G}(r), k}(x)=\operatorname{rdet}_{(k)}\left(x I_{n}-\Gamma_{G}(r)\right)=\lambda_{0}^{(k)}+\lambda_{1}^{(k)} x+\cdots+\lambda_{n^{k}-1}^{(k)} x^{n^{k}-1}+\lambda_{n^{k}}^{(k)} x^{n^{k}},
$$

$\lambda_{n^{k}}^{(k)}=q \geq 1$ is an integer and $\lambda_{i}^{(k)} \in \operatorname{Fix}(\tau)$ for each $0 \leq i \leq n^{k}$. Thus we have

$c_{i}=\frac{1}{q} \lambda_{i}^{(k)} \in \operatorname{Fix}(G)$ for each index $0 \leq i \leq n^{k}-1$. It follows that

$$
\begin{gathered}
\Gamma_{G}\left(c_{0}+r c_{1}+\cdots+r^{n^{k}-1} c_{n^{k}-1}+r^{n^{k}}\right)= \\
I_{n} c_{0}+\Gamma_{G}(r) c_{1}+\cdots+\left(\Gamma_{G}(r)\right)^{n^{k}-1} c_{n^{k}-1}+\left(\Gamma_{G}(r)\right)^{n^{k}}= \\
\left(I_{n} \lambda_{0}^{(k)}+\Gamma_{G}(r) \lambda_{1}^{(k)}+\cdots+\left(\Gamma_{G}(r)\right)^{n^{k}-1} \lambda_{n^{k}-1}^{(k)}+\left(\Gamma_{G}(r)\right)^{n^{k}} \lambda_{n^{k}}^{(k)}\right) \cdot \frac{1}{q}=0
\end{gathered}
$$

and $\operatorname{ker}\left(\Gamma_{G}\right)=\{0\}$ gives the desired right integrality.

Proof of Theorem (B). Consider the embeddings

$$
\Delta: R \longrightarrow \mathrm{M}_{n}(R, \delta, H) \text { and } \Delta^{(\delta)}: R[w, \delta] \longrightarrow \mathrm{M}_{n}\left(R[z], \delta_{z}, H\right)
$$

in Corollary 2.4, where $\Delta(r)=\sum_{i=1}^{n} \delta^{i}(r) E_{i, i}, H=E_{1,2}+E_{2,3}+\cdots+E_{n-1, n}+E_{n, 1}$ and

$$
\Delta^{(\delta)}\left(r_{0}+r_{1} w+\cdots+r_{t} w^{t}\right)=\Delta\left(r_{0}\right)+\Delta\left(r_{1}\right) H z+\cdots+\Delta\left(r_{t}\right) H^{t} z^{t} .
$$

Since $R[z]$ is also Lie nilpotent of index $k$, Theorem 4.5 gives that

$$
\begin{gathered}
I_{n} \lambda_{0}^{(k)}(z)+\Delta^{(\delta)}(f(w)) \lambda_{1}^{(k)}(z)+\cdots \\
\cdots+\left(\Delta^{(\delta)}(f(w))^{n^{k}-1} \lambda_{n^{k}-1}^{(k)}(z)+\left(\Delta^{(\delta)}(f(w))^{n^{k}} \lambda_{n^{k}}^{(k)}(z)=0,\right.\right.
\end{gathered}
$$

where the coefficients of the $k$-th right characteristic polynomial

$\operatorname{rdet}_{(k)}\left(x I_{n}-\Delta^{(\delta)}(f(w))\right)=\lambda_{0}^{(k)}(z)+\lambda_{1}^{(k)}(z) x+\cdots+\lambda_{n^{k}-1}^{(k)}(z) x^{n^{k}-1}+\lambda_{n^{k}}^{(k)}(z) x^{n^{k}}$ of the $\left(\delta_{z}, H\right)$-centralizing matrix $\Delta^{(\delta)}(f(w)) \in \mathrm{M}_{n}\left(R[z], \delta_{z}, H\right)$ are in $\operatorname{Fix}\left(\delta_{z}\right)=$ $\operatorname{Fix}(\delta)[z]$ and $\lambda_{n^{k}}^{(k)}(z)=q \geq 1$ is an integer.

According to Example (2), the natural $\mathbb{Z}_{n}$-grading

$$
R[z]=R\left[z^{n}\right] \oplus z R\left[z^{n}\right] \oplus \cdots \oplus z^{n-1} R\left[z^{n}\right]
$$

of the polynomial algebra $R[z]$ defines $\mathrm{M}_{n}^{g}(R[z])$ as

$$
\mathrm{M}_{n}^{g}(R[z])=\left\{A=\left[a_{i, j}(z)\right] \in \mathrm{M}_{n}(R[z]) \mid a_{i, j}(z) \in z^{j-i} R\left[z^{n}\right] \text { for all } 1 \leq i, j \leq n\right\}=
$$




$$
\left[\begin{array}{ccccc}
R\left[z^{n}\right] & z R\left[z^{n}\right] & \cdots & z^{n-2} R\left[z^{n}\right] & z^{n-1} R\left[z^{n}\right] \\
z^{n-1} R\left[z^{n}\right] & R\left[z^{n}\right] & z R\left[z^{n}\right] & \ddots & z^{n-2} R\left[z^{n}\right] \\
\vdots & z^{n-1} R\left[z^{n}\right] & \ddots & \ddots & \vdots \\
z^{2} R\left[z^{n}\right] & \ddots & \ddots & R\left[z^{n}\right] & z R\left[z^{n}\right] \\
z R\left[z^{n}\right] & z^{2} R\left[z^{n}\right] & \cdots & z^{n-1} R\left[z^{n}\right] & R\left[z^{n}\right]
\end{array}\right]=\mathrm{M}_{n}\left(R[z], \widehat{e}, D_{e}\right) .
$$

Clearly, $\Delta\left(r_{i}\right) \in \mathrm{M}_{n}^{g}(R[z])$ and $H z \in \mathrm{M}_{n}^{g}(R[z])$ imply that $\Delta\left(r_{i}\right) H^{i} z^{i} \in \mathrm{M}_{n}^{g}(R[z])$ for all $i \geq 0$. Thus we have $\Delta^{(\delta)}(f(w)) \in \mathrm{M}_{n}^{g}(R[z])$ for all $f(w) \in R[w, \delta]$. The use of Corollary 4.4 gives that

$$
\operatorname{rdet}_{(k)}\left(x I_{n}-\Delta^{(\delta)}(f(w))\right) \in\left(R\left[z^{n}\right]\right)[x],
$$

whence $\lambda_{i}^{(k)}(z)=\mu_{i}\left(z^{n}\right) \in \operatorname{Fix}(\delta)\left[z^{n}\right]$ follows for all $0 \leq i \leq n^{k}-1$.

Since $H^{n}=I_{n}$, for any polynomial $\mu\left(z^{n}\right) \in \operatorname{Fix}(\delta)\left[z^{n}\right]$ there exists a (unique) polynomial $g\left(w^{n}\right) \in \operatorname{Fix}(\delta)\left[w^{n}\right]$ such that

$$
\begin{gathered}
\mu\left(z^{n}\right) I_{n}=c_{0} I_{n}+c_{1} z^{n} I_{n}+\cdots+c_{d} z^{n d} I_{n}= \\
\Delta\left(c_{0}\right)+\Delta\left(c_{1}\right) H^{n} z^{n}+\cdots+\Delta\left(c_{d}\right) H^{n d} z^{n d}= \\
\Delta^{(\delta)}\left(c_{0}+c_{1} w^{n}+\cdots+c_{d} w^{n d}\right)=\Delta^{(\delta)}\left(g\left(w^{n}\right)\right),
\end{gathered}
$$

where the coefficients $c_{0}, c_{1}, \ldots, c_{d} \in \operatorname{Fix}(\delta)$ of $\mu\left(z^{n}\right)$ and $g\left(w^{n}\right)$ coincide. For each $0 \leq i \leq n^{k}-1$ take $g_{i}\left(w^{n}\right) \in \operatorname{Fix}(\delta)\left[w^{n}\right]$ such that

$$
\Delta^{(\delta)}\left(g_{i}\left(w^{n}\right)\right)=\frac{1}{q} \mu_{i}\left(z^{n}\right) I_{n}=\frac{1}{q} \lambda_{i}^{(k)}(z) I_{n} .
$$

Thus

$$
\begin{gathered}
\Delta^{(\delta)}\left(g_{0}\left(w^{n}\right)+f(w) g_{1}\left(w^{n}\right)+\cdots+f^{n^{k}-1}(w) g_{n^{k}-1}\left(w^{n}\right)+f^{n^{k}}(w)\right)= \\
\frac{1}{q}\left(I_{n} \lambda_{0}^{(k)}(z)+\Delta^{(\delta)}(f(w)) \lambda_{1}^{(k)}(z)+\cdots\right. \\
\cdots+\left(\Delta^{(\delta)}(f(w))^{n^{k}-1} \lambda_{n^{k}-1}^{(k)}(z)+\left(\Delta^{(\delta)}(f(w))^{n^{k}} \lambda_{n^{k}}^{(k)}(z)\right)=0\right.
\end{gathered}
$$

and $\operatorname{ker}\left(\Delta^{(\delta)}\right)=\{0\}$ gives the desired integrality.

\section{REFERENCES}

[Do] M. Domokos, Cayley-Hamilton theorem for $2 \times 2$ matrices over the Grassmann algebra, J. Pure Appl. Algebra 133 (1998), 69-81.

[Ke] A. R. Kemer, Ideals of Identities of Associative Algebras, Translations of Math. Monographs, Vol. 87 (1991), AMS, Providence, Rhode Island.

[S1] J. Szigeti, New determinants and the Cayley-Hamilton theorem for matrices over Lie nilpotent rings, Proc. Amer. Math. Soc. 125 (1997), 2245-2254.

[S2] J. Szigeti, On the characteristic polynomial of supermatrices, Israel Journal of Mathematics Vol. 107 (1998), 229-235.

[SvW] J. Szigeti and L. van Wyk, Determinants for $n \times n$ matrices and the symmetric Newton formula in the $3 \times 3$ case, Linear and Multilinear Algebra, Vol. 62, No. 8 (2014), 1076-1090.

Institute of Mathematics, University of Miskold, Miskold, Hungary 3515

E-mail address: matjeno@uni-miskolc.hu 